\title{
The role of chemokines and cytokines in lung fibrosis
}

\author{
M.P. Keane
}

\begin{abstract}
Idiopathic pulmonary fibrosis (IPF) exhibits a complex and poorly understood pathogenesis. Overt inflammation in the lungs of patients with established IPF is absent, and classic anti-inflammatory therapies are inefficacious; however, inflammation may contribute to the disruption of the normal alveolar architecture, allowing interaction between fibroblasts and the epithelium. The polarisation of the inflammatory response toward a type-2 helper T-cell phenotype may also be important in the development of pulmonary fibrosis, as fibroproliferation could be favoured over repair. Furthermore, evidence has emerged regarding an imbalance between angiogenic and angiostatic chemokine levels, leading to an overall angiogenic pattern of expression in both animal models and tissue specimens from IPF patients. The precise role of vascular remodelling in IPF remains to be determined. Therefore, numerous questions exist regarding the role and importance of chemokines and cytokines in pulmonary fibrosis. Further investigation is required to facilitate the elucidation of IPF pathogenesis and identification of novel targets for treatment of this dismal disease.
\end{abstract}

KEYWORDS: Angiogenesis, idiopathic pulmonary fibrosis, inflammation

\section{INTRODUCTION}

\section{Type-1 and type-2 T-cell response}

The differentiation between type- 1 and type- 2 helper T-cell (Th1 and Th2) responses in humans is not as straightforward as it is in mouse models. Profiles of cytokines that fit these patterns are known to exist, however, which suggests that the polarisation of the inflammatory responses they represent may be important in the development of fibrosis. The Th1 response includes increased expression of interferon (IFN) $-\gamma$ and interleukin (IL)-2, IL-12 and IL-18, all of which support cellmediated immunity and in general promote tissue restoration. In contrast, the classic Th2 cytokines, IL-4, IL-5, IL-10 and IL-13, are more involved with antibody-mediated immunity, and tend to promote fibroblast activation, matrix development and, therefore, fibrosis (fig. 1).

Certain chemokine receptors predominate on either Th1 or Th2 cells. In terms of Th1 cells, these include CXCR3, which is expressed on lymphocytes and on endothelial cells and also has been shown to have a beneficial role in terms of limiting fibrosis as, discussed hereunder. Cytokine receptors expressed by Th2 cells include CC chemokine receptor (CCR)4, CCR8 and CXCR4. There are data from experimental models that support the importance of the Th2 response in pulmonary fibrosis. CC chemokine ligand (CCL)17 and CCL22 are associated with a Th2 profile and are significantly elevated in bleomycin-induced pulmonary fibrosis [1]. In addition, their receptor CCR4 is significantly elevated in bleomycin-treated mice, predominantly on macrophages. Neutralisation of CCL17 leads to a reduction in pulmonary fibrosis in this model Immunolocalisation of CCL17 in bleomycin-treated and human lung tissue specimens from patients with idiopathic pulmonary fibrosis (IPF) shows that it is predominantly expressed on epithelial cells (fig. 2) [1]. CCL22 levels are also raised in lung tissue from patients with IPF, and CCL22 is predominantly expressed on epithelium, particularly hyperplastic epithelium.

These findings lend some support to a type-2 inflammatory response promoting fibrosis and indicate a connection between this response and epithelial cells, the primary site of damage in IPF. It is possible to hypothesise that, at some time before the patient presents, an initial epithelial injury stimulates an acute inflammatory response and release of early response cytokines. In the "normal" situation, the inflammatory response may polarise toward a Th1-type response, with inhibition of the Th2 response leading to stability or resolution of the lung injury.

If a Th2 response develops, this could lead to a robust fibroproliferative response and the progressive scarring and fibrosis seen in IPF.
CORRESPONDENCE

M.P. Keane

University College Dublin

St Vincent's University Hospital Elm Park

Dublin 4

Ireland

Fax: 35312693587

E-mail: michael.p.keane@ucd.ie

STATEMENT OF INTEREST

M.P. Keane has received fees for participating in an advisory board for Actelion. 


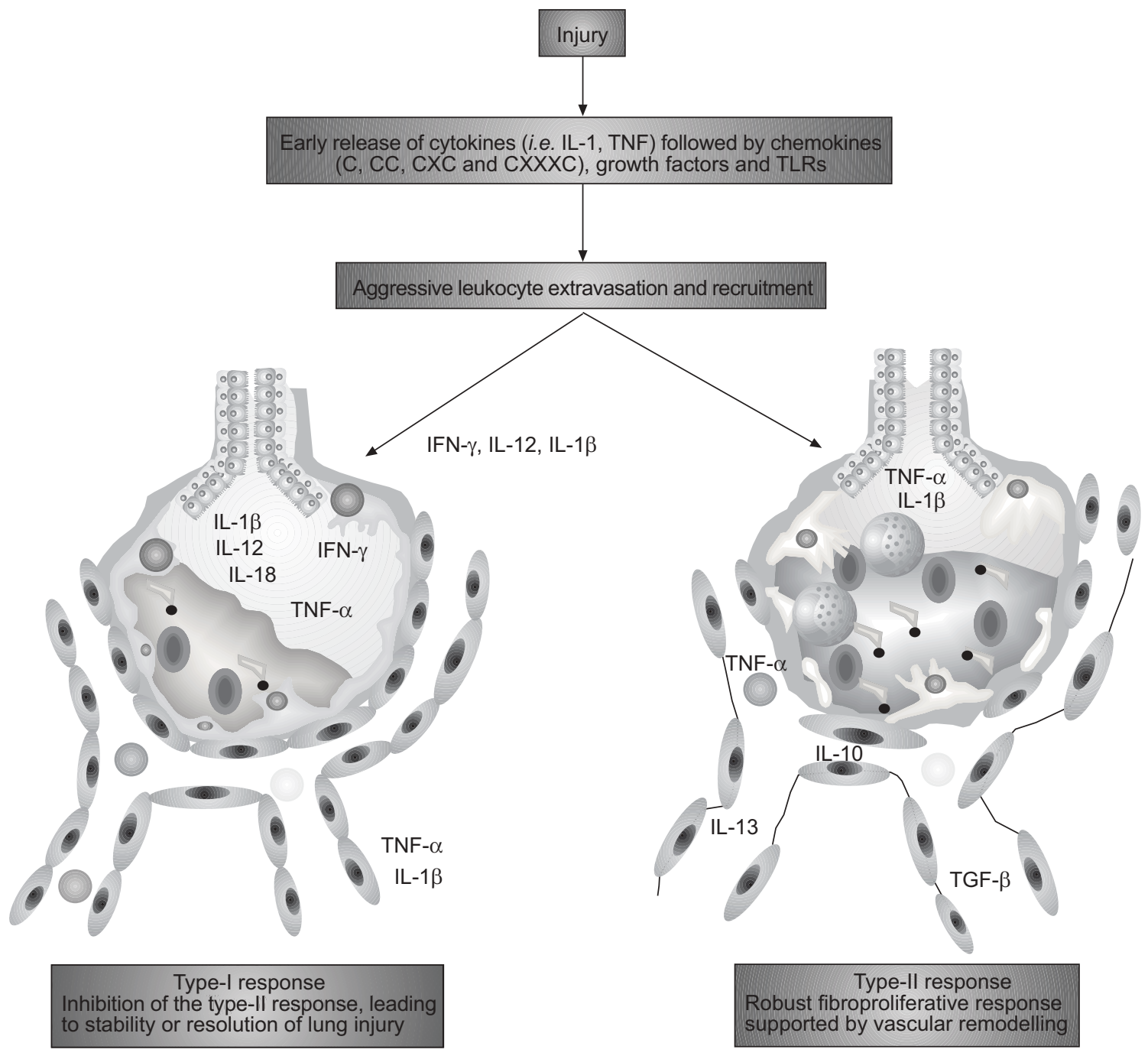

FIGURE 1. An illustration of type-I and type-II T-cell responses arising from alveolar injury. IL: Interleukin; TNF: tumour necrosis factor; TLR: Toll-like receptor IFN: interferon; TGF: transforming growth factor.

Some evidence against this model is the lack of effectiveness of the prototypic type- 1 cytokine, IFN- $\gamma$, which has been shown to have no effect on IPF in a large-scale clinical trial [2]. In reality, it is unlikely that the inflammatory response in IPF fits neatly into these two categories or is so polarised, although consideration of this model and the various cytokines and chemokines involved may help to define the process leading to fibrosis in these patients.

\section{Bronchiolitis obliterans syndrome as a model of the continuum of inflammation and fibrosis}

Bronchiolitis obliterans syndrome after lung transplantation is characterised by progressive airway obstruction associated with fibrosis of the small airways [3]. The histological pattern varies depending on when lung tissue is examined, with marked inflammatory infiltration in the early, acute phase and extensive fibrosis without obvious infiltration at the end stage of disease [4]. This model demonstrates that, although inflammation may not be present in the later stages of the disease, it clearly plays an important role in its pathogenesis. Therefore, the absence of inflammation reported in IPF does not necessarily rule out a potential role at an early stage before patients present. However, by the time they do present, inflammation is certainly less prominent, which probably accounts for the lack of efficacy of current anti-inflammatory regimens.

\section{Macrophages and fibrosis}

Macrophages are an important source of growth factors, cytokines and metalloproteases involved in fibrosis. An early study showed that the degree of staining of CD60-positive interstitial macrophages correlated with the degree of clinical impairment and collagen deposition in patients with IPF [5]. As in the Th1/Th2 system, macrophages also show two polarised phenotypes termed M1 and M2 cells, which are selected by microenvironmental factors. Classically, M1 macrophages are induced by IFN- $\gamma$ alone or in combination with microbial factors, such as lipopolysaccharide, or cytokines, including tumour necrosis factor (TNF) and 

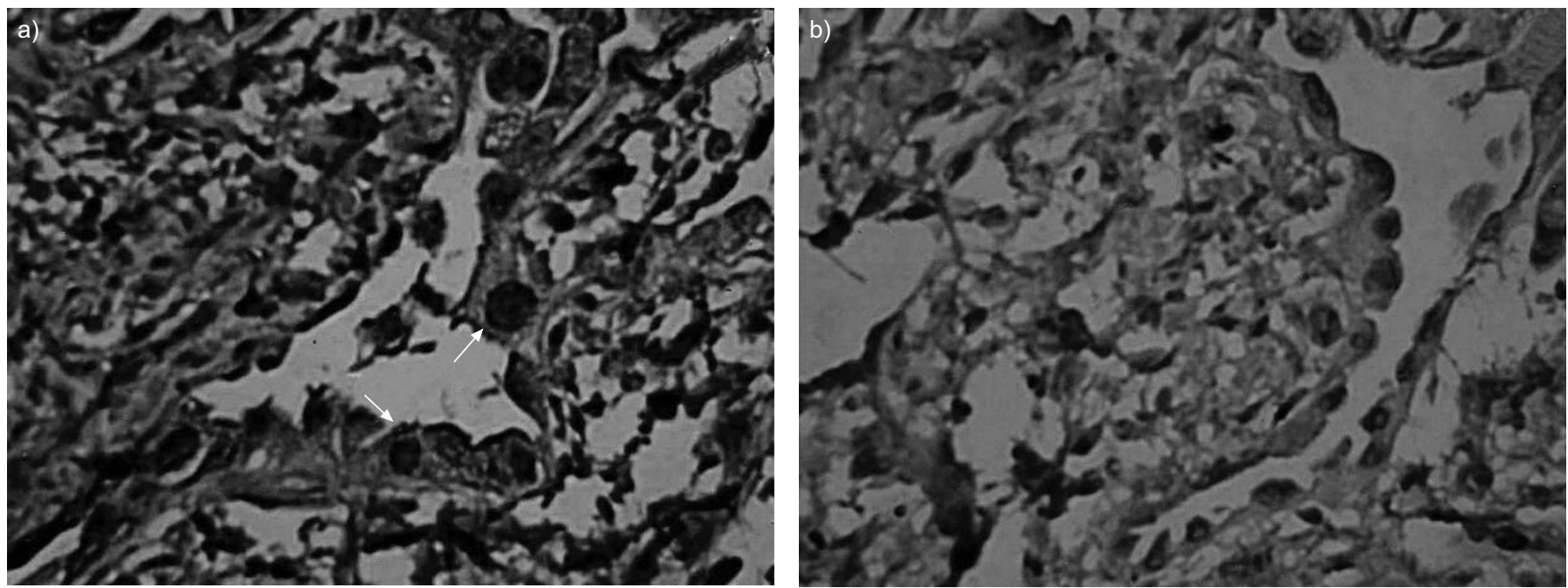

FIGURE 2. Photomicrograph showing immunolocalisation of CC chemokine ligand (CCL) 17 from idiopathic pulmonary fibrosis (IPF) lung tissue specimens. a) IPF lung section stained with anti-CCL17 antibodies, demonstrating immunolocalisation to epithelial cells. b) IPF lung section stained with control antibodies, demonstrating the lack of nonspecific staining. Reproduced with from [1] with permission from the publisher.

granulocyte-macrophage colony-stimulating factor [6]. The alternative M2 form of macrophage activation is mediated by a range of factors, including IL-4 or IL-13, immune complexes and IL-10. M1 macrophages promote strong IL-12-mediated Th1 responses and are proficient producers of effector molecules (reactive oxygen and nitrogen intermediates) and inflammatory cytokines (IL-1 $\beta$, TNF- $\alpha$, IL-6), whereas M2 cells support Th2 effector functions, and are also proposed to have a role in the control of cell growth and collagen deposition [7]. Therefore, the macrophage is an inflammatory cell that potentially has a role to play in the pathogenesis of IPF, and deserves further investigation.

\section{Lymphoid neogenesis in IPF}

Ectopic formation of secondary lymphoid tissue has been linked to chronic inflammation in mouse models and human diseases, and has also been demonstrated in IPF [8]. Interestingly, these lymphoid aggregates did not contain CCR7-positive naïve Tcells, proliferating lymphocytes or germinal centres, suggesting that antigen-experienced lymphocytes and newly recruited mature dendritic cells can drive lymphoid neogenesis. Lymphadenopathy is a relatively common finding on computed tomography (CT) scans of patients with IPF, and tends to be dismissed as a reactive finding. Whether there is an association between lymphadenopathy and lymphoid neogenesis or whether this is just an epi-phenomenon remains to be established.

\section{Implications for therapy}

Despite the fact that corticosteroids have been considered the mainstay of IPF treatment for decades, there is a lack of conclusive evidence supporting their efficacy [9]. Although other anti-inflammatory agents have been used, it is generally recognised that conventional anti-inflammatory therapy has very little role in the management of IPF [9]. However, this lack of responsiveness may be a reflection of the nonspecific nature of current anti-inflammatory agents, e.g. steroids inhibit both Th1 and Th2 responses and also limit prostaglandin E2, which would actually be beneficial as it prevents fibroblast proliferation and collagen production. It is possible that, if antiinflammatory agents are to be of any use at all, more discriminatory regimens are needed, although given the apparent lack of inflammation when patients with IPF present, it may be that even this strategy is too late and that inflammation at that time may not be a very important target.

TABLE 1 Structural and functional differences of ELR-positive CXC chemokines in the regulation of vascular remodelling

Chemokine Effect on angiogenesis Relevant structural motif $\quad$ Receptor through which effects on angiogenesis
are mediated

\begin{tabular}{llll}
\hline CXCL1 & Angiogenic & A-T-E-L-R-C-Q-C & CXCR2 \\
CXCL2 & Angiogenic & A-T-E-L-R-C-Q-C & CXCR2 \\
CXCL3 & Angiogenic & V-T-E-L-R-C-Q-C & CXCR2 \\
CXCL5 & Angiogenic & L-R-E-L-R-C-V-C & CXCR2 \\
CXCL8 & Angiogenic & A-K-E-L-R-C-Q-C & CXCR2 \\
\hline
\end{tabular}

ELR motif: a conserved amino acid sequence of Glu-Leu-Arg in the amino terminus of CXC chemokines. Reproduced from [11] with permission from the publisher 
TABLE 2 Structural and functional differences of ELR-negative CXC chemokines in the regulation of vascular remodelling

Chemokine

Effect on angiogenesis

Relevant structural motif

Receptor through which effects on angiogenesis

are mediated

\begin{tabular}{llll}
\hline CXCL4 & Angiostatic & D-G-D-L-Q-C-L-C & CXCR3 \\
CXCL9 & Angiostatic & V-R-K-G-R-C-S-C & CXCR3 \\
CXCL10 & Angiostatic & S-R-T-V-R-C-T-C & CXCR3 \\
CXCL11 & Angiostatic & F-K-R-G-R-C-L-C & CXCR3
\end{tabular}

ELR motif: a conserved amino acid sequence of Glu-Leu-Arg in the amino terminus of CXC chemokines. Reproduced from [11] with permission from the publisher.

\section{VASCULAR REMODELLING AND ANGIOGENESIS IN IPF}

Vascular remodelling in IPF was originally identified over 40 yrs ago by TURNER-WARWICK [10], who demonstrated extensive vascular remodelling in the lungs of patients with widespread interstitial fibrosis leading to anastomoses between the systemic and pulmonary microvasculature. These sites of remodelling were observed to correlate with the subpleural regions where fibrosis would be more marked. The role this remodelling plays still remains to be determined.

Further studies have found that the bronchoalveolar lavage fluid (BALF) and lung tissue from patients with IPF exhibit marked angiogenic activity that is almost entirely attributable to overexpression of angiogenic chemokines and the relative downregulation of angiostatic chemokines.

The family of CXC motif chemokines is unique in that different family members regulate vascular remodelling in a disparate manner [11]. Immediately preceding the conserved CXC motif is a second structural domain that dictates their functional activity in regulating angiogenesis. Several family members contain a three amino acid sequence (Glutamate-Leucine-Arginine), known as the ELR motif. ELR-positive CXC chemokines promote angiogenesis (table 1). CXC cytokines lacking the ELR motif (ELR-negative) are IFN-inducible and inhibit angiogenesis (table 2). The difference in response to ELRpositive and ELR-negative CXC chemokines results from their binding to different CXC chemokine receptors (CXCRs) on endothelial cells; ELR-positive CXC cytokines bind to CXCR2, whereas ELR-negative CXC chemokines bind to CXCR3.
In IPF there is increased angiogenic activity in the BALF. Various groups have shown that the levels of the ELR-positive chemokines CXCL5 and CXCL8 are increased, and levels of ELR-negative chemokines CXCL10 and CXCL11 are decreased, leading to an imbalance that tends to favour an angiogenic response $[12,13]$. In a murine model of bleomycin-induced pulmonary fibrosis, extensive neovascularisation has often been observed to follow the airways and the sites of the injury [14]. The amounts of CXCL2 and CXCL3, and of CXCL10 and CXCL11, in the lung during bleomycin-induced pulmonary fibrosis have been found to be directly and inversely correlated, respectively, with measures of fibrosis [15, 16]. Administration of the ELR-negative CXCL10 [16] or CXCL11 [17] to these animals during exposure to bleomycin attenuates pulmonary fibrosis by a process that is attributable to reduced angiogenesis.

Further evidence for the importance of chemokines and their receptors in pulmonary fibrosis comes from CXCR3- and CXCL10-deficient mice models. Mice deficient for CXCR3 show increased mortality and progressive interstitial fibrosis relative to controls [18]. They demonstrate a decreased expression of CXCL10 and a reduced early burst of IFN- $\gamma$ production following lung injury, suggesting that the innate production of IFN $-\gamma$ may be important in limiting fibrosis [18]. CXCL10 is highly expressed in a mouse model of pulmonary fibrosis induced by bleomycin. Mice deficient in CXCL10 show increased pulmonary fibrosis after administration of bleomycin, while transgenic mice overexpressing CXCL10 exhibited decreased fibrosis and mortality after bleomycin exposure [19].
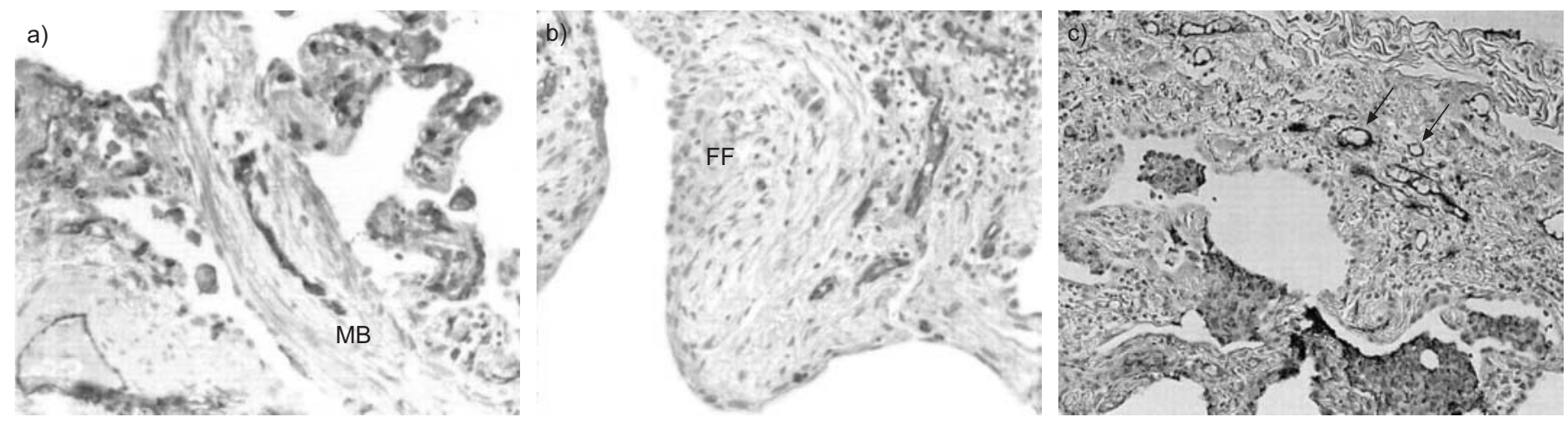

FIGURE 3. Immunohistochemistry for CD31 counterstained with haematoxylin, demonstrating positive staining in a) organising pneumonia and b) usual interstitial pneumonia (UIP). c) Within structurally distorted areas in UIP, termed honeycomb lung, abnormal, large-diameter vessels were apparent (arrows). FF: fibroblastic foci; MB Masson bodies. Reproduced from [20] with permission from the publisher. 


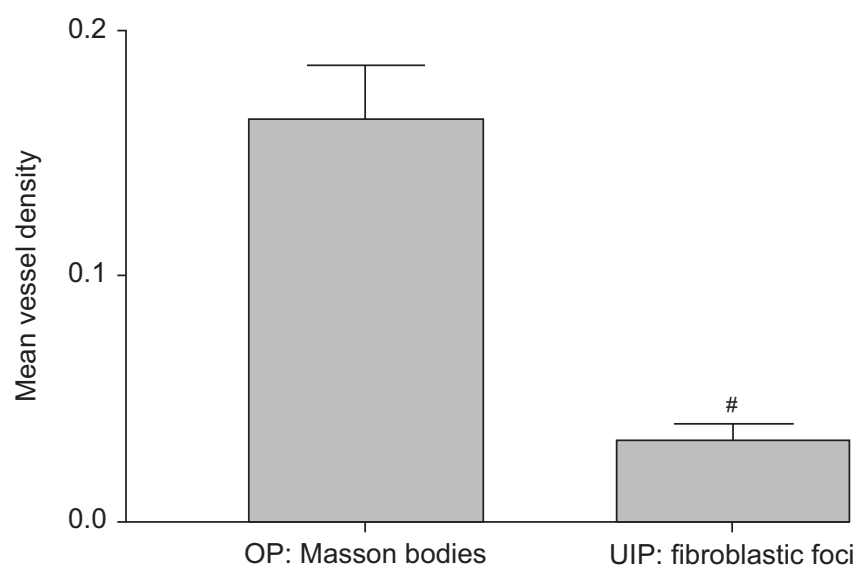

FIGURE 4. Semiquantitative immunohistochemical analysis revealed a significant difference in CD31 positivity (mean \pm SEM vascular density in organising pneumonia (OP) Masson bodies $(n=25) 0.163 \pm 0.022$; in usual interstitial pneumonia (UIP) fibroblastic foci $(n=30) 0.032 \pm 0.007 ; p=0.015)$, suggesting a difference in vascularisation between the Masson bodies of cryptogenic organising pneumonia and the fibroblastic foci of UIP. ${ }^{*}: p=0.015$ by unpaired t-test. The mean vessel density is the amount of CD31 positivity normalised with respect to area. Reproduced from [20] with permission from the publisher

The levels of CXCL10 expression were associated with the degree of fibroblast accumulation seen following bleomycin exposure, with a dramatic increase in CXCL10-deficient mice and a decrease in CXCL10 transgenic mice, compared with wildtype controls. These data suggest an important role for CXCL10/ CXCR3 in the development of fibrosis, at least in the mouse model, although they did not indicate an effect on angiogenesis.

More evidence for a potential role of angiogenesis in the pathogenesis of IPF has come from work on pigment epithelium-derived factor (PEDF), a 50-kD protein with angiostatic and neurotrophic activities that regulates vascular development in the eye. COSGROve et al. [20] found that PEDF expression was increased in the lungs of patients with IPF based on microarray analyses. They also found regional decreased vascularity within fibroblastic foci that correlated with increased PEDF expression and decreased expression of vascular endothelial growth factor. Surrounding these foci were areas of honeycombing that showed dilated and abnormal vasculature (fig. 3). PEDF was also shown to colocalise with transforming growth factor (TGF)- $\beta$ and, furthermore, PEDF was shown to be a TGF- $\beta 1$ target gene. Together these findings implicate PEDF as a regulator of pulmonary angiogenesis and a potential mediator of fibrosis in IPF [20]. Interestingly, COSGROve et al. [20] also compared cryptogenic organising pneumonia (COP) and found that, in contrast to IPF, there was increased vascularity within fibrotic foci, and that the amount of vasculature was significantly different between these two diseases (fig. 4). Whether these differences in vascularity play a role in the greater responsiveness of COP to therapy compared with that of IPF remains to be determined. Similar findings of decreased vascularity in fibroblastic foci in fibrotic lung disease and increased abnormality of the vasculature surrounding these foci have also been reported by other groups [21, 22].
The question that arises is whether the degree of vascular remodelling seen in IPF is appropriate. There is certainly regional heterogeneity with regard to vascular remodelling, which is seen whether CT scans or biopsies are examined. There is no doubt that there is an abnormal vasculature in the lungs of patients with IPF, including pulmonary systemic anastomosis, which may have a role in the refractory hypoxaemia seen in some of these patients.

\section{CONCLUSIONS}

Despite the lack of overt inflammation in the lungs of patients with established disease, the inflammatory response may still play an early role in the pathogenesis of IPF. Inflammation may set the stage for fibrosis in IPF and may contribute to the disruption of the normal alveolar architecture, allowing interaction between fibroblasts and epithelium, and it may result in the activation of profibrotic Th2 and M2 phenotypes. The presence of lymphoid neogenesis in IPF is interesting and evocative, although its true role remains to be determined. The possibility that the inflammatory response may be important in the initiation of the pathogenesis of IPF, and the potential complexities of that response, may explain the lack of efficacy of current anti-inflammatory regimens. Indiscriminate antiinflammatory agents may equally affect beneficial Th1-type cytokines as well as potentially detrimental Th2-type cytokines. More discriminatory regimens may be helpful, but it may prove to be that, by the time patients present, even targeted anti-inflammatory therapies will be insufficient.

Evidence suggests that the balance between angiogenic and angiostatic CXC chemokines is important in the management and control of vascular remodelling and angiogenesis. Given the close association between angiogenesis and fibrotic disorders, an imbalance in these various cytokine profiles may be associated with the development of fibrosis. This raises a number of questions regarding the importance of vascular remodelling in idiopathic pulmonary fibrosis. For example, does it vary with the stage of the disease? Is it just an epiphenomenon or is it directly involved in the pathogenesis? Does it, perhaps, contribute to hypoxia? Might it contribute to the vascular remodelling that leads to pulmonary hypertension, which is increasingly recognised in around one-third of patients with idiopathic pulmonary fibrosis [23]? Importantly, does it also have implications for the access of systemic treatments to poorly vascularised fibrotic foci or their overlying epithelium? Answering some of these questions may help elucidate the pathogenesis of idiopathic pulmonary fibrosis and may lead to novel targets for therapies for this dismal disease.

\section{REFERENCES}

1 Belperio JA, Dy M, Murray L, et al. The role of the Th2 CC chemokine ligand CCL17 in pulmonary fibrosis. J Immunol 2004; 173: 4692-4698.

2 Raghu G, Brown KK, Bradford WZ, et al. A placebocontrolled trial of interferon $\gamma-1 b$ in patients with idiopathic pulmonary fibrosis. N Engl J Med 2004; 350: 125-133.

3 Nicod LP. Mechanisms of airway obliteration after lung transplantation. Proc Am Thorac Soc 2006; 3: 444-449. 
4 Myers JL, Colby TV. Pathologic manifestations of bronchiolitis, constrictive bronchiolitis, cryptogenic organizing pneumonia, and diffuse panbronchiolitis. Clin Chest Med 1993; 14: 611-622.

5 Uh ST, Inoue Y, King TE Jr, Chan ED, Newman LS, Riches DW. Morphometric analysis of insulin-like growth factor-I localization in lung tissues of patients with idiopathic pulmonary fibrosis. Am J Respir Crit Care Med 1998; 158: 1626-1635.

6 Martinez FO, Gordon S, Locati M, Mantovani A. Transcriptional profiling of the human monocyte-tomacrophage differentiation and polarization: new molecules and patterns of gene expression. J Immunol 2006; 177: 7303-7311.

7 Shearer JD, Richards JR, Mills CD, Caldwell MD. Differential regulation of macrophage arginine metabolism: a proposed role in wound healing. Am J Physiol 1997; 272: E181-E190.

8 Marchal-Sommé J, Uzunhan Y, Marchand-Adam S, et al. Cutting edge: nonproliferating mature immune cells form a novel type of organized lymphoid structure in idiopathic pulmonary fibrosis. Immunol 2006; 176: 5735-5739.

9 Bouros D, Antoniou KM. Current and future therapeutic approaches in idiopathic pulmonary fibrosis. Eur Respir J 2005; 26: 693-702.

10 Turner-Warwick M. Precapillary systemic-pulmonary anastomoses. Thorax 1963; 18: 225-37.

11 Strieter RM, Gomperts BN, Keane MP. The role of CXC chemokines in pulmonary fibrosis. J Clin Invest 2007; 117: 549-556.

12 Keane MP, Arenberg DA, Lynch JP 3rd, et al. The CXC chemokines, IL-8 and IP-10, regulate angiogenic activity in idiopathic pulmonary fibrosis. J Immunol 1997; 159: 1437-1443.

13 Antoniou KM, Tzouvelekis A, Alexandrakis MG, et al. Different angiogenic activity in pulmonary sarcoidosis and idiopathic pulmonary fibrosis. Chest 2006; 130: 982-988.
14 Peao MN, Aguas AP, de Sa CM, Grande NR. Neoformation of blood vessels in association with rat lung fibrosis induced by bleomycin. Anat Rec 1994; 238: 57-67.

15 Keane MP, Belperio JA, Moore TA, et al. Neutralization of the CXC chemokine, macrophage inflammatory protein-2, attenuates bleomycin-induced pulmonary fibrosis. J Immunol 1999; 162: 5511-5518.

16 Keane MP, Belperio JA, Arenberg DA, et al. IFN- $\gamma-$ inducible protein-10 attenuates bleomycin-induced pulmonary fibrosis via inhibition of angiogenesis. J Immunol 1999; 163: 5686-5692.

17 Burdick MD, Murray LA, Keane MP, et al. CXCL11 attenuates bleomycin-induced pulmonary fibrosis via inhibition of vascular remodeling. Am J Respir Crit Care Med 2005; 171: 261-268.

18 Jiang D, Liang J, Hodge J, et al. Regulation of pulmonary fibrosis by chemokine receptor CXCR3. J Clin Invest 2004; 114: 291-299.

19 Tager AM, Kradin RL, LaCamera P, et al. Inhibition of pulmonary fibrosis by the chemokine IP-10/CXCL10. Am J Respir Cell Mol Biol 2004; 31: 395-404.

20 Cosgrove GP, Brown KK, Schiemann et al. Pigment epithelium-derived factor in idiopathic pulmonary fibrosis: a role in aberrant angiogenesis. Am J Respir Crit Care Med 2004; 170: 242-251.

21 Renzoni EA, Walsh DA, Salmon M, et al. Interstitial vascularity in fibrosing alveolitis. Am J Respir Crit Care Med 2003; 167: 438-443.

22 Ebina M, Shimizukawa M, Shibata N, et al. Heterogeneous increase in CD34-positive alveolar capillaries in idiopathic pulmonary fibrosis. Am J Respir Crit Care Med 2004; 169: 1203-1208.

23 Lettieri CJ, Nathan SD, Barnett SD, Ahmad S, Shorr AF. Prevalence and outcomes of pulmonary arterial hypertension in advanced idiopathic pulmonary fibrosis. Chest 2006; 129: 746-752. 